Case Report

\title{
Premature Ventricular Contractions (PVCs) Induced by Administration of Cilostazol after Myocardial Infarction
}

\author{
Lihua Zhong ${ }^{1}$, Yanhui Gao ${ }^{1}$, Xueqi Li ${ }^{1}$, Shipeng Wei ${ }^{1,2}$ \\ ${ }^{1}$ The 4th Clinical Hospital of Harbin Medical University, Harbin, China \\ ${ }^{2}$ University of Alabama at Birmingham, Birmingham, USA
}

Received 31 July 2012, Revised 31 October 2012, Accepted 20 November 2012.

(C) 2012, Zhong L., Gao Y., Li X., Wei S.

(C) 2012, Russian Open Medical Journal

\begin{abstract}
Cilostazol is a phosphodiesterase III inhibitor, which was approved by FDA (food and drug administration) for intermittent claudication in 1999. What made it known to cardiologists is the Cilostazol for Restenosis Trial (CREST), in which cilostazol was shown to significantly lower binary restenosis rate. Here we reported one case of acquired premature ventricular contractions (PVCs) during taking cilostazol. A 68-year-old male patient was diagnosed acute myocardial infarction and given percutaneous coronary intervention (PCI). It turned out there was thrombi in the distal right coronary artery. The patient was discharged taking cilostazol (100mg Bid) together with aspirin and clopidogrel. On follow-up, there were PVCs coming up (more than 27000 beats in 24-hour electrocardiography) and cilostazol was discontinued afterwards. After taking metoprolol $(25 \mathrm{mg}$, Tid) for one week, the PVCs were decreased significantly, 600 beats/24 hours. In our case, the PVCs are obviously related to cilostazol and probably the safety of cilostazol in patients having a history of myocardial infarction or malignant arrhythmias should be reevaluated.
\end{abstract}

Keywords: premature ventricular contractions (PVCs), cilostazol, phosphodiesterase.

Cite as Zhong L, Gao Y, Li X, Wei S. Premature ventricular contractions (PVCs) induced by administration of cilostazol after myocardial infarction. Russian Open Medical Journal 2013; 2: 0105.

Correspondence to Shipeng Wei. Address: Department of Cardiology, the 4th Clinical Hospital of Harbin Medical University, 37 Yiyuan Street, Nangang District, Harbin, 150001, China. Tel: +86 (451) 82579351. E-mail: Shipengwei@yahoo.com.

\section{Introduction}

Cilostazol was approved by FDA (food and drug administration) in 1999 for intermittent claudication. What made it known to cardiologists is the Cilostazol for Restenosis Trial (CREST) [1]. In recent years, it was found out that cilostazol had many other uses. Koji and his colleagues reported that cilostazol was effective and safe to be applied in patients with three-degree atrioventricular block for a short-term [2]. Cilostazol was also reported to reduce ST segment elevation, thus acting to diminish the action potential notch and suppress the substrate and trigger for ventricular tachycardia/fibrillation (VT/VF), which made it a candidate for brugada syndrome treatment [3]. The CREST (Cilostazol for Restenosis Trial) released in 2005, showed that administration of cilostzaol together with aspirin and clopidogrel significantly lower binary restenosis rate [1]. It seems that cilostazol is a promising drug, while still based on the mechanism, it showed some sideeffects, like ventricular arrhythmias, haemorrhage, interaction with other drugs or food and many more. In our case, A 68-yearold male patient diagnosed acute myocardial infarction without histories of arrythmias developed a severe premature ventricular contractions (PVCs) while taking cilostazol and the PVCs decreased significantly after cilostazol discontinuation. So the PVCs are obviously related to cilostazol and probably the safety of cilostazol in patients having a history of myocardial infarction or malignant arrhythmias should be reevaluated.

\section{Case presentation}

General history: A 68-year-old male patient, complaining persistent chest pain for 18 hours, was admitted to our hospital.

Previous medical history: totally 5 coronary stents were implanted in 2007 and 2008, respectively; there are no histories of drinking or smoking.

Physical examination: Respiratory rate: 18 times/min; heart rate: 84 beats/min; blood pressure: 120/76 $\mathrm{mmHg}$. No other positive signs were detected.

\section{Auxiliary Examinations:}

Electrocardiography (ECG): Sinus rhythm, heart rate: 84 beats/min; In II, III, AVF leads, ST segments elevation, diminished R waves and abnormal $Q$ waves were seen.

Echocardiography: Left atrium: $39 \mathrm{~mm}$, left ventricle: $49 \mathrm{~mm}$, ejection fraction: $58 \%$.

\section{Blood test:}

$\mathrm{K}+: 3.67 \mathrm{mmol} / \mathrm{L} ; \mathrm{Na}+: 142.2 \mathrm{mmol} / \mathrm{L} ; \mathrm{Cl}-: 105.8 \mathrm{mmol} / \mathrm{L} ; \mathrm{Ca}++$ : $2.08 \mathrm{mmol} / \mathrm{L}$; UREA: $5.1 \mathrm{mmol} / \mathrm{L} ; \quad A S T: 155 \mathrm{U} / \mathrm{L} ; \mathrm{CK}: 1624 \mathrm{U} / \mathrm{L}$; CKMB: 151 U/L; LDH: 385 U/L; HBDH: 348 U/L; TBIL: 26.3 ummo/L; others were normal. 


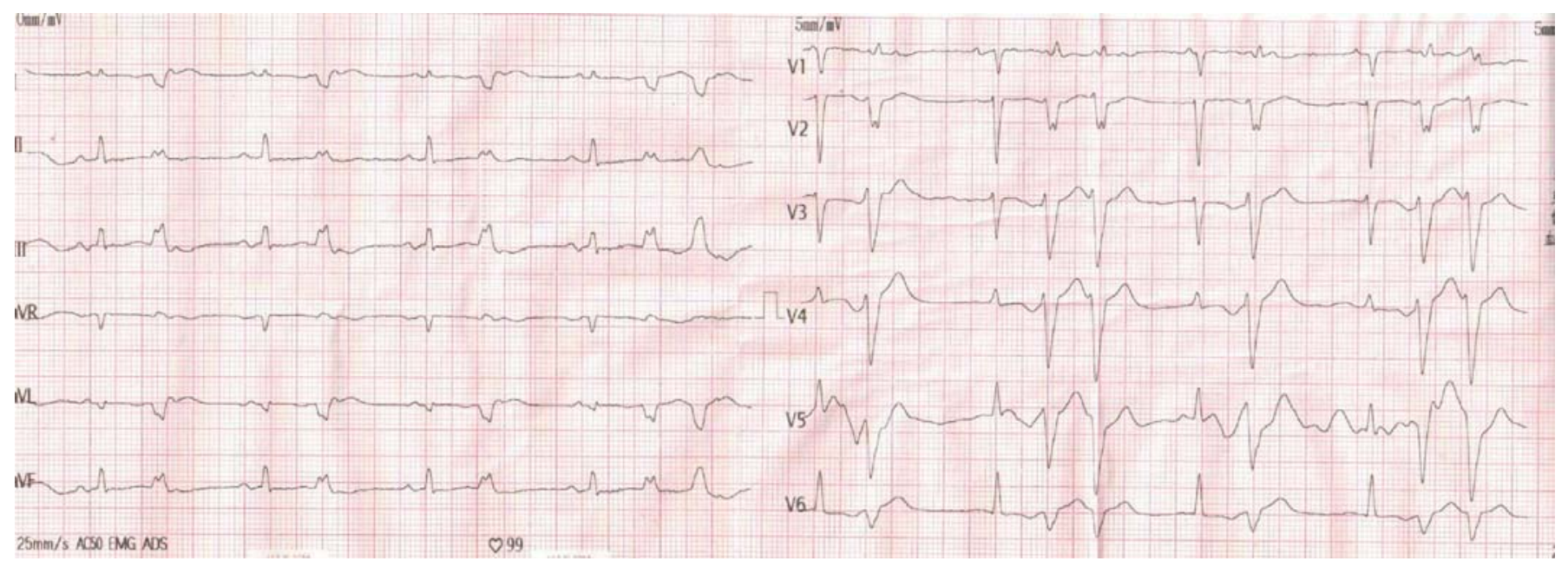

Figure 1. ECG after oral cilostazol for one week showed premature ventricular contractions (PVCs), more than 27000 beats/24 hours.
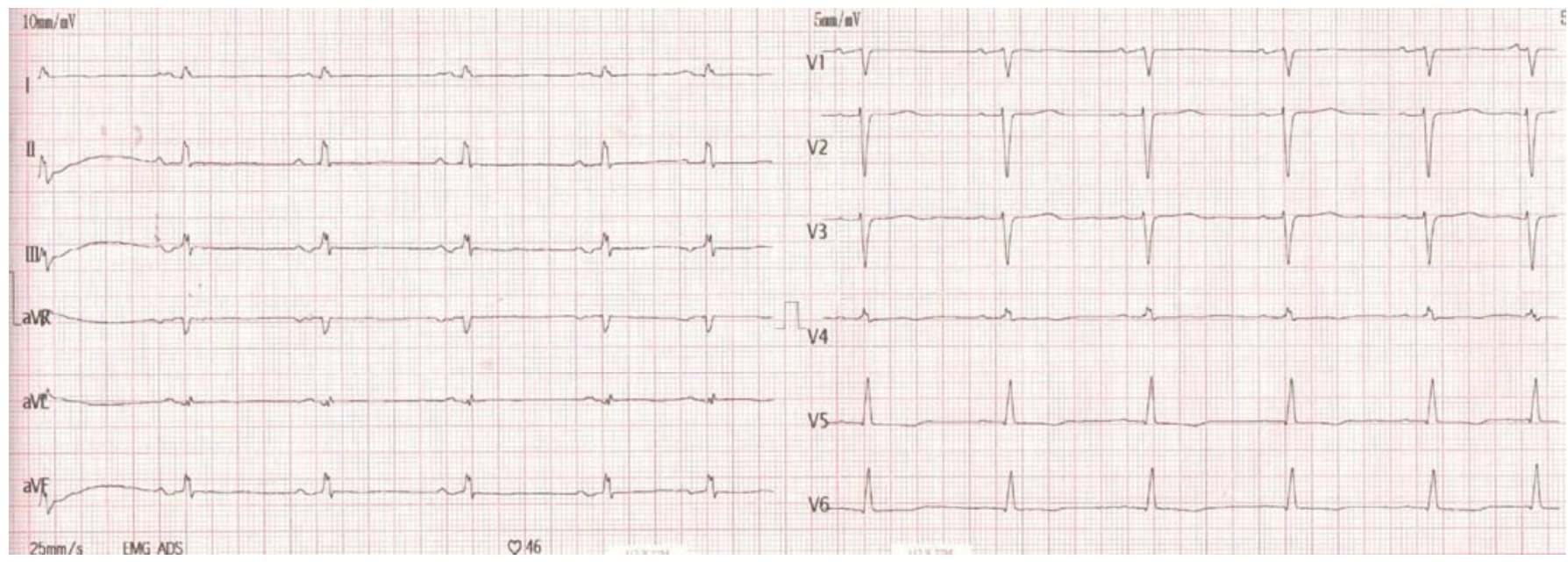

Figure 2. ECG one week after discontinuation of cilostazol showed PVCs, 600beats/24 hours.

Aspirin, clopidogrel and atorvastatin were administrated and immediate coronary angiography was performed, which showed thrombi in the distal right coronary artery. After given tirofiban, the thrombi was dissolved and the patient was discharged with prescription of oral cilostazol (100 mg, Bid). While one week later, he felt palpitation and 24-hour ECG showed PVCs, more than 27000 beats/24 hours (Figure 1). Cilostazol was discontinued and metoprolol (25 mg, Tid) was applied. 24-hour ECG after one week showed PVCs, 600 beats/24 hours (Figure 2).

\section{Discussion}

Cilostazol, a phosphodiesterase (PDE) III inhibitor, which was first approved in Japan and 3 years later in the US is long used for intermittent claudication [4]. Based on its mechanism and tissue specifities, it mainly affect cardiac monocytes, vascular smooth vessels and platelets, therefore cilostazol is far more than just an antithrombotic agent.

Cilostazol is widely used in the treatment of three-degree atrioventricular block, Brugada syndrome and post percutaneous coronary intervention [1-3]. CREST (Cilostazol for Restenosis Trial) released in 2005, first showed that administration of cilostzaol together with aspirin and clopidogrel significantly lower binary restenosis rate without increasing major complications [1].
Cilostazol was also administrated in high risk patients implanted with drug-eluting stents [5]. Some Korean colleagues used cilostazol in patients having acute myocardial infarction (AMI) and still an exciting result was achieved [6]. It is thought a promising drug with little side effects till recent years, different voices came up. It was reported that cilostazol increased the incidence of ventricular arrhythmias and the mean number of episodes in infarcted animals. Barta et al found the same thing not only in infarcted but also in normal animals under epinephrine challenged test [7]. These bring up a question, whether it is worthwhile to take the risk having cilostazol? While it was not significant in the CREST [1], so further studies should be carried out to evaluate the safety of cilostazol in infracted patients.

In our case, cilostazol is a compensatory way to treat coronary thrombi. During taking cilostazol, the patient developed PVCs. Since he didn't have any histories of arrhythmias and after discontinuation of cilostazol, the PVCs decreased significantly, the arrhythmia is highly associated with cilostazol. It made us feel a little nervous that every year ventricular arrhythmias kills thousands of patients having acute myocardial infarction (AMI) throughout the world. And clinical drugs either offer exogenous or enhance endogenous CAMP are forbidden in AMI patients. CREST showed a decrease in binary restenosis in patients receiving stents without differences in bleeding, rehospitalization, target-vessel 
revascularization, myocardial infarction, or death [1]. While the patients recruited in this study are with stable angina or unstable angina or silent myocardial ischemia. So further studies including broad spectrum of patients should be carried out. Even though, we still should be careful to apply cilostazol in patients having a history of myocardial infarction or malignant arrhythmias.

\section{Acknowledgement}

We thank Lili Mao who collected all the information of the patients.

\section{Conflicts of Interest}

All authors don't have conflicts of interest.

\section{Reference}

1. Douglas JS Jr, Holmes DR Jr, Kereiakes DJ, Grines CL, Block E et al. Coronary Stent Restenosis in Patients Treated With Cilostazol. Circulation 2005; 112: 2826-32. (doi: 10.1161/ CIRCULATIONAHA.104.530097)

2. Kodama-Takahashi K, Kurata A, Ohshima K, Yamamoto K, Uemura S, Watanabe S, Iwata T. Effect of Cilostazol on the Ventricular Escape Rate and Neurohumoral Factors in Patients With Third-Degree Atrioventricular Block. Chest 2003; 123: 1161-9. (PMID: 12684307) (doi: 10.1378/chest.123.4.1161)

3. Shimlizul W, Aiba T, Antzelevitch C. Specific Therapy Based on the Genotype and Cellular Mechanism in Inherited Cardiac Arrhythmias. Long QT Syndrome and Brugada Syndrome. Curr Pharm Des 2005; 11(12): 1561-72. (PMID: 15892662) (doi: 10.2174/1381612053764823)

4. Reilly MP, Mohler ER 3rd. Cilostazol: treatment of intermittent claudication. Ann Pharmacol 2001; 35: 48-56. (PMID: 11197586)

5. Lee SW, Chun KJ, Park SW, Kim HS, Kim YH, Yun SC, Kim WJ et al. Comparison of Triple antiplatelet therapy and dual antiplatelet therapy in patients at high risk of restenosis after drug-eluting stent implantation (from the DECLARE-DIABETES and -LONG Trials). Am J Cardiol 2010; 105(2): 168-73. (doi: 10.1016/j.amjcard.2009.08.667) (PMID: 20102913)

6. Jeong $\mathrm{YH}, \mathrm{Hwang} \mathrm{JY}$, Kim IS, Park Y, Hwang SJ, Lee SW, Kwak CH, Park SW. Adding cilostazol to dual antiplatelet therapy achieves greater platelet inhibition than high maintenance dose clopidogrel in patients with acute myocardial infarction: Results of the adjunctive cilostazol versus high maintenance dose clopidogrel in patients with $\mathrm{AMI}$ (ACCEL-AMI) study. Circ Cardiovasc Interv 2010; 3(1): 17-26. (doi: 10.1161/CIRCINTERVENTIONS.109.880179) (PMID: 20118150)

7. Barta J, Sanganalmath SK, Kumamoto H, Takeda N, Edes I, Dhalla NS. Antiplatelet agents sarpogrelate and cilostazol affect experimentallyinduced ventricular arrhythmias and mortality. Cardiovasc Toxicol 2008; 8(3): 127-35. (doi: 10.1007/s12012-008-9019-x) (PMID: 18751928)

Authors:

Lihua Zhong - MD, Associate Professor, Department of Cardiology, The 4th Clinical Hospital of Harbin Medical University, Harbin, 150001, China;

Yanhui Gao - MD, Senior Resident, Department of Cardiology, The 4th Clinical Hospital of Harbin Medical University, Harbin, 150001, China;

Xueqi Li - MD, Professor, Department of Cardiology, The 4th Clinical Hospital of Harbin Medical University, Harbin, 150001, China;

Shipeng Wei - MD, Assistant Professor, Department of Cardiology, The 4th Clinical Hospital of Harbin Medical University, Harbin, 150001, China; Department of Cell, Developmental and Integrative Biology, University of Alabama at Birmingham, Birmingham, AL 35294, USA. 